\title{
IMPAIRED NEURODEVELOPMENTAL OUTCOME ASSOCIATED WITH INCREASED WHITE MATTER CHO/CR IN PRETERM INFANTS
}

\author{
G.S. Kendall ${ }^{1}$, D. Price ${ }^{2}$, A. Bainbridge ${ }^{2}$, S. Johnson ${ }^{1,3}$, C. Hagmann ${ }^{1}$, R. Gunny ${ }^{4}$, N. Marlow ${ }^{1}$, E.B. Cady ${ }^{2}$, \\ N.J. Robertson ${ }^{1}$ \\ ${ }^{I}$ Academic Neonatology, University College London, ${ }^{2}$ Medical Physics \& Bio-Engineering, University \\ College London Hospital NHS Foundation Trust, ${ }^{3}$ Department of Health Sciences, University of Leicester, \\ ${ }^{4}$ Neuroradiology, Great Ormond Street Hospital for Children NHS Trust, London, UK
}

Background: Diffuse white matter (WM) injury is the commonest MR finding in preterm infants. We aimed to describe the metabolic signature of diffuse WM injury and correlate to neurodevelopmental outcome at 1 year.

Methods: 45 preterm infants $(<32$ weeks) underwent single WM voxel point-resolved spectroscopy (fig1A\&B) at mean corrected gestational age (CGA) of $40 \pm 1.6$ weeks. Metabolite ratios were quantified using AMARES algorithm. Bayley III was performed at a corrected age of 1 year. Infants with scores < $1 \mathrm{SD}$ in each domain classified as impaired.

Results: Only Naa/Cho correlated to gestation at birth $(\mathrm{r}=0.39 \mathrm{p}<0.05) .1$ year outcome data was available for $42 / 45$. Mean cognitive $(96 \pm 14)$ and motor $(87 \pm 15)$ composite scores were within the normative population range. Motor outcomes significantly correlated with both $\mathrm{Naa} / \mathrm{Cho}$ and $\mathrm{Cho} / \mathrm{Cr}$ ratios before and after correction for gestational age (table 1). After excluding babies with brain abnormalities, there was a significant increase in $\mathrm{Cho} / \mathrm{Cr}$ in the motor impaired group $(\mathrm{p}<0.05)$ (fig1C).

Conclusions: Localised white matter proton $\mathrm{MRS} \mathrm{Cho} / \mathrm{Cr}$ at term corrected age may provide a useful biomarker of motor outcome in preterm infants.
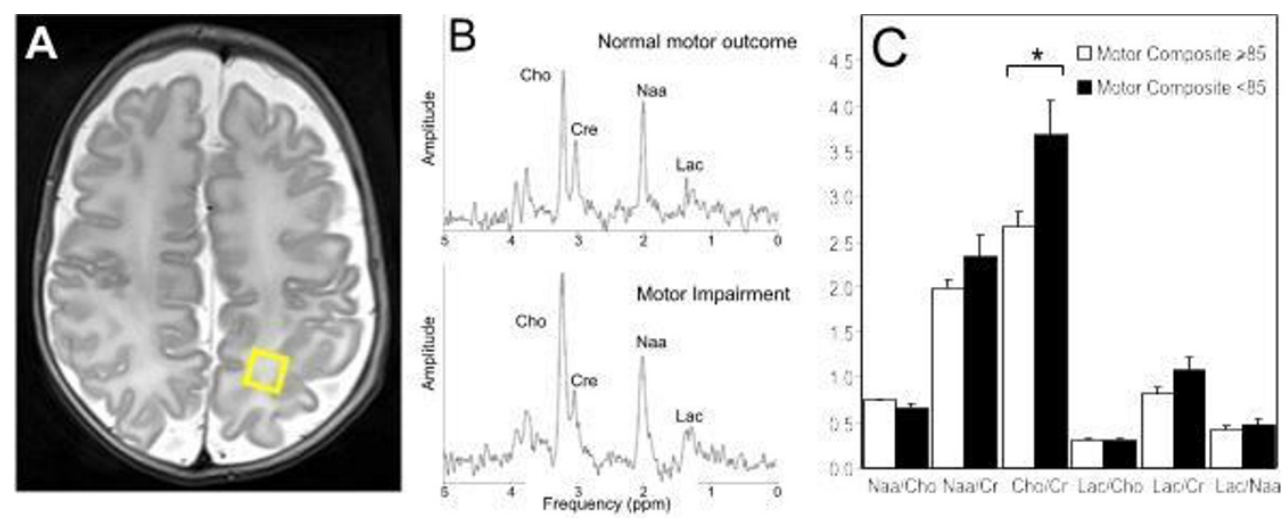

Figure 1: (A) The MRS voxel positioned in left PWM on an axial image. (B) Representative $1 \mathrm{H}$ spectra from babies with and without motor impairment. (C) Metabolite ratios in babies with and without motor impairment at 1 year corrected PN age.

[Figure1]

\begin{tabular}{|c|c|c|c|c|c|c||}
\hline \multicolumn{1}{|c|}{} & \multicolumn{3}{c|}{ Raw Correlation } & \multicolumn{2}{c|}{ Corrected for Gestational Age } \\
\hline & Gross Motor & Fine Motor & Composite & Gross Motor & Fine Motor & Composite \\
\hline \hline Naa/Cho & $\mathrm{r}=0.44, \mathrm{p}=0.004$ & $\mathrm{r}=0.41, \mathrm{p}=0.007$ & $\mathrm{r}=0.47, \mathrm{p}=0.002$ & $\mathrm{r}=0.38, \mathrm{p}=0.015$ & $\mathrm{r}=0.32, \mathrm{p}=0.041$ & $\mathrm{r}=0.39, \mathrm{p}=0.011$ \\
\hline Cho/Cre & $\mathrm{r}=0.49, \mathrm{p}=0.002$ & $\mathrm{r}=0.32, \mathrm{p}=0.040$ & $\mathrm{r}=0.41, \mathrm{p}=0.007$ & $\mathrm{r}=0.42, \mathrm{p}=0.007$ & $\mathrm{r}=0.24, \mathrm{p}=0.124$ & $\mathrm{r}=0.349, \mathrm{p}=0.026$ \\
\hline [Table1] & & & & \\
\hline
\end{tabular}

